\title{
EXPERIENCES WITH PREVENTING CARPAL TUNNEL SYNDROME IN AN AUTOMOTIVE PLANT
}

\section{VĚRA ŽÍDKOVÁ', MARIE NAKLÁDALOVÁ1, JANA ZAPLETALOVÁ2 ZDENĚK NAKLÁDAL ${ }^{3}$, and HELENA KOLLÁROVÁ ${ }^{4}$}

${ }^{1}$ Palacký University, Olomouc, Czech Republic

Department of Occupational Medicine

${ }^{2}$ Palacký University, Olomouc, Czech Republic

Department of Medical Biophysics

${ }^{3}$ Regional Public Health Authority of the Olomouc Region, Olomouc, Czech Republic

${ }^{4}$ Palacký University, Olomouc, Czech Republic

Department of Preventive Medicine

\begin{abstract}
Objectives: Carpal tunnel syndrome (CTS) is a common occupational disease. The aim was to assess the effect of preventive measures in automotive assembly workers. Material and Methods: The analysis summarizes data from annual crosssectional studies. The 7-year analysis of data was based on medical records obtained from an occupational physician and inspections carried out at the workplace where targeted preventive measures were introduced, including better ergonomic arrangement of the workplace, technical adjustments facilitating the work, preventive nerve conduction studies (NCS) testing of the median nerve once a year, switching of workers and their targeted rotation within the workplace. The NCS testing of median nerve conduction at the wrist was the basic objective method for assessment of the prevalence and severity of CTS. Over the study period, the sample comprised 1804 workers at risk for repetitive overuse of the upper extremities, of whom 281 were females with a mean age of 38.5 years and 1523 were males with a mean age of 31.4 years. Results: Over the study period, a total of 13 cases of CTS were recognized as an occupational disease in the plant, 8 of which occurred within the first 2 years from the initiation of production. Introduction of preventive measures decreased the prevalence of median neuropathy from $18.3 \%$ of examined extremities in 2011 to $10.5 \%$ in 2013 ( $p=0.003$ ). In early 2014, the production pace increased and this was accompanied by a rise in abnormal NCS findings to $16.9 \%$. Over the study period, the rate of sensorimotor neuropathy decreased in favor of merely sensory neuropathies, which have been most frequent since 2013. The percentage of employees whose contracts were terminated due to median neuropathy decreased steadily from $5.5 \%$ to $0.4 \%$. Conclusions: Targeted prevention of work-related CTS is effective as evidenced by the decrease in the prevalence of median neuropathy detected by NCS. Int J Occup Med Environ Health 2017;30(1):45-54
\end{abstract}

Key words:

Risk factors, Occupational diseases, Prevention, Carpal tunnel syndrome, Nerve conduction studies, Repetitive overuse

Funding: the article was supported through long-term conceptual development research organization with identification No. 61989592 - Palacký University, Olomouc, Czech Republic.

Received: September 10, 2015. Accepted: January 11, 2016.

Corresponding author: V. Žídková, Palacký University, Department of Occupational Medicine, I.P. Pavlova 6, 77520 Olomouc, Czech Republic (e-mail: lekar.baska@seznam.cz). 


\section{INTRODUCTION}

Carpal tunnel syndrome (CTS) is the most common neuropathy of the upper extremity, accounting for more than $90 \%$ of all nerve entrapment syndromes [1-5].

The carpal tunnel is a fibro-osseous structure made up of carpal bones bridged over by the transverse carpal ligament. Apart from the median nerve, 9 flexor tendons of the hand pass through it. The median nerve consists of both sensory and motor fibers.

Clinical manifestations of the condition are both irritation (particularly paresthesia, most frequently of the thumb, index finger, middle finger and, partly, of the ring finger) and, in advanced stages, deficits (weakness or atrophy of the thenar muscle, decreased muscle strength, clumsiness of the hand, dropping things). Initially, these difficulties occur when the hand is rested for a long time, especially at night; they gradually worsen, occurring in daytime as well, and become permanent. Their intensity varies among individuals and may fluctuate with time in the same person [6].

Similarly, neurological clinical examination including provocative diagnostic maneuvers often gives positive results only in more severe conditions. Therefore, the basic objective exact method for diagnosing CTS including its initial forms is nerve conduction study (NCS). In the Czech Republic, standard methods have been established that include the procedure and conditions for electromyography (EMG) to determine the severity of isolated CTS [7].

In addition to common risk factors such as age, gender, obesity, thyroid disease, hormone replacement therapy, diabetes mellitus or mental stress, occupation also plays a role in the development of CTS [1,8-13]. The prevalence of CTS is significantly higher in manual workers than in the general population. Specific jobs cannot be accurately defined, but manual labor generally is considered a risk factor. Assembly workers in the automotive industry have traditionally been frequently affected.
In both 2013 and 2014, this was the single most affected job in the Czech Republic [14]. In many studies, CTS has been associated with the following risk factors: repetitive wrist movement, chronic wrist flexion, firm grip on tools or long-term exposure to vibrations. The dominant risk factor is repetitive overuse of the upper extremity $[13,15]$.

The fact that the work performed by a worker may result in CTS is reflected by legislation in many countries, among others by recognizing the condition as an occupational disease with relevant financial compensations.

In the European Union, the condition has been listed as an occupational disease since 2003; in Germany, it has been recognized as an occupational disease since July 2009 [2]. In the Czech Republic, CTS due to upper extremity overuse has been recognized as an occupational disease since as early as 1975 [16]. Recognition of the condition as an occupational disease in the Czech Republic is based on strict criteria [17]. In the last 10 or more years, CTS due to upper extremity overuse has been the most frequently reported occupational disease in the Czech Republic [14].

The article summarizes 7-year experience with occupational health care for male and female automotive assembly workers exposed to repetitive overuse and monitors trends in the prevalence of CTS in the context of preventive measures introduced, thus attempting to assess their benefit. The basic method for objective assessment of CTS and its severity in the present study was NCS testing.

\section{MATERIAL AND METHODS}

The study analyzed the prevalence of median neuropathy detected by NCS testing, with regard to preventive measures being introduced, in the period from the initiation of production in January 2008 until the end of 2014 in automotive assembly workers. The data were processed in the form of cross-sectional studies related 
to individual calendar years between 2008 and 2014 . The data analysis was based on preventive health examinations performed by a single occupational physician over the entire study period. The basic objective method for assessing both the prevalence and severity of CTS was an annual NCS test of the median nerve introduced in the plant's assembly workers in 2010. In that year, however, only $20 \%$ of the employees were examined. Therefore, the statistics include NCS results from 2011-2014, when a mean of $79.7 \%$ of all assembly line workers performing the job for longer than 1 year were examined annually.

All NCS examinations were carried out in the same electrophysiological laboratory by a single neurologist using the standard methods, that is, median sensory conduction velocity (SCV) to the index or middle finger at $14 \mathrm{~cm}$ and median distal motor latency (DML) at $8 \mathrm{~cm}$ [7].

To assess conduction of impulses in sensory fibers, the basic parameter was SCV; to determine conduction of impulses in motor fibers, DML was used.

The results of NCS testing were evaluated as follows: normal median conduction study ( $\mathrm{SCV}>50 \mathrm{~m} / \mathrm{s}$ and $\mathrm{DML}<4.2 \mathrm{~ms}$ ). Median nerve damage of medium severity met the following conditions: $\mathrm{SCV} \leq 38 \mathrm{~m} / \mathrm{s}$ and DML $\geq 5.3 \mathrm{~ms}$; in the Czech Republic, such severity is the criterion for recognizing CTS as an occupational disease. Exact criteria for medium severity of NCS findings are specified in the methodology description [7]. Mild median neuropathy was characterized by the following values: $\mathrm{SCV}=38.1-40 \mathrm{~m} / \mathrm{s}$ and $\mathrm{DML}=4.9-5.29 \mathrm{~ms}$; under certain circumstances, such nerve damage may be a prerequisite for the so-called risk of occupational disease in the Czech Republic. If the finding was less severe than mild but not in the normal range, it was labeled as incipient median neuropathy for the purposes of this study; the other abnormal NCS findings were considered as advanced neuropathy.

\section{Sample and working conditions}

The sample comprised all assembly line workers exposed to repetitive overuse (category $2 \mathrm{R}$ ). This category includes work activities in which hygiene limits are not exceeded but there is a certain risk of work-related health damage [18]. In the plant, these activities were assembling engines, gearboxes, cabling, superchargers, chassis, stabilizer bars, shock absorbers, brake discs, pads and other components. The activities were carried out by standing workers using their upper extremities and mainly handling small parts such as bolts or light loads $<10 \mathrm{~kg}$. The employees worked manually, only rarely and irregularly using pneumatic torque wrenches.

Given the fact that for operational reasons, the assembly line operators' work could not be organized in such a way that they did not perform repetitive activities leading to upper extremity overuse, the employer provided them with health and safety breaks in accordance with Czech legislation from the very beginning of production [19]. In addition to meal breaks, the employees had two 10-min health and safety breaks included in their 8-h shifts. The occurrence of occupational diseases prompted a search for effective preventive measures. Through cooperation between an occupational physician, the company's health and safety at work specialist and the company management, several preventive measures have been introduced since 2010, including:

- An ergonomic arrangement of the workplace was introduced in 2010 (Photo 1). To the maximum extent possible, the operator's workplace was adapted so that parts and tools were more effectively arranged to reduce the range of motion of the upper extremities and the entire body. Lifting aids and tool holders were installed and parts to be assembled (bolts, gaskets, belts) were placed in containers within the natural reach of the hand; similarly, controllers were placed at the level of the hand. Anti-stress mats were placed on the floor or under the feet 
and the workplaces were equipped with drink bottle holders.

- Since 2010, all exposed workers have undergone NCS testing performed once a year and paid for by the company. The aim is to detect individuals with incipient median nerve neuropathy at the wrist so that the trend of NCS findings may be studied. In case of a significant progression of the condition, the worker was shifted to a different position where the load on the upper extremities was lower; in extreme cases, the workers were excluded from the risk of overuse.

- In 2011, a strict switching of workers and their targeted rotation within the same workplace were introduced, aimed at changing the movement stereotypes and load distribution. There is a 2-h switching pattern with electronic records; an operator has to clock in to the workplace using an electronic identification card and his/her position may be found in a computer database.

The employees' working speed had remained practically unchanged from the initiation of production until

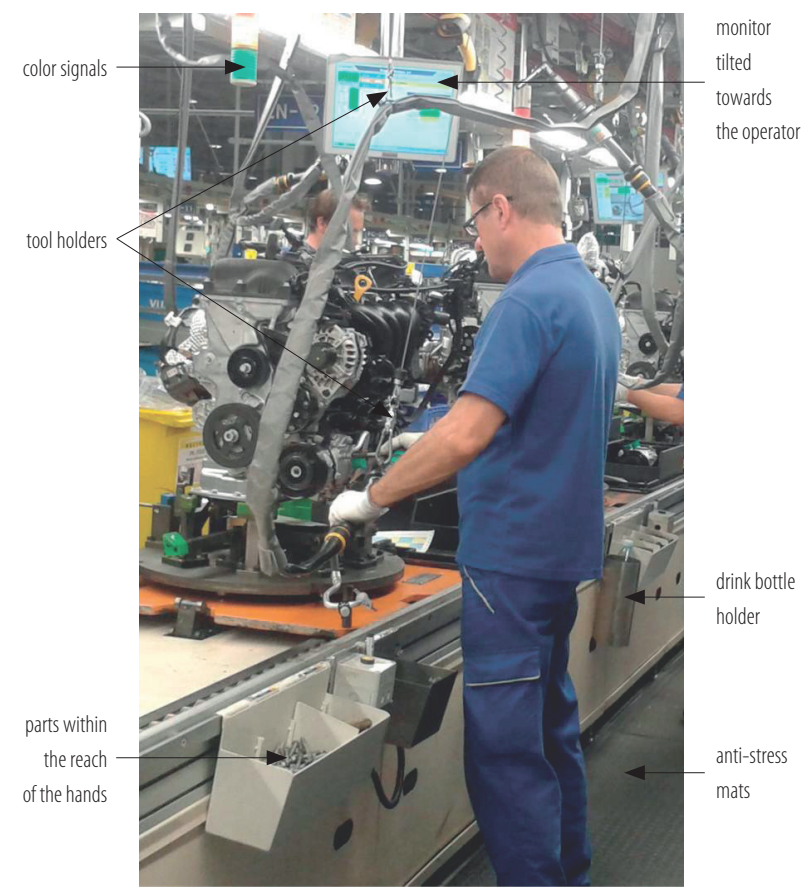

Photo 1. Assembly line worker in automotive plant early 2014 when the production pace increased due to a growing demand.

The number of employees fluctuated from year to year as the automotive industry dynamically evolved, flexibly reflecting the market demand, as documented by Table 1 . The overall number of employees working in the plant for various periods of time was 1804, of whom 281 were females and 1523 were males. On average, 308 assembly line workers were employed in the plant each year, comprising 69 females with a mean age of 38.5 years (range: $20-58$ years, median $(\mathrm{Me})=39$ years) and 239 males with a mean age of 31.4 years (range: 18-63 years, $\mathrm{Me}=41$ years). Given the fact that the study aimed at assessing the effectiveness of preventive measures introduced in the plant, only a subgroup of employees working there for at least 1 year was selected for a detailed analysis.

The data were statistically processed with the IBM SPSS Statistics. A year-to-year comparison was conducted using Fisher's exact test with Bonferroni correction of significance.

\section{RESULTS}

Over the study period, CTS due to repetitive overuse was recognized as an occupational disease in a total of 13 individuals working in the plant, with a mean age of 44.8 years $(\mathrm{Me}=44$ years $)$ and a mean exposure of 21.4 months ( $\mathrm{Me}=27$ months). The condition was recognized in 4 males with a mean age of 45.5 years (range: $20-57$ years, $\mathrm{Me}=52.5$ years) and a mean exposure of 27 months (range: 6-46 months, $\mathrm{Me}=21.5$ months) and in 9 females with a mean age of 44.4 years (range: $39-56$ years, $\mathrm{Me}=43$ years) and a mean exposure of 20.3 months (range: 6-30 months, $\mathrm{Me}=27$ months).

The occurrence of CTS as an occupational disease in workers at risk for repetitive overuse over the study period is shown in Table 2. 
Table 1. Characteristics of the respondents at risk for repetitive overuse of the upper extremities - working in automotive plant, Czech Republic, 2011-2014

\begin{tabular}{lllll}
\hline \multicolumn{1}{c}{ Respondents } & \multicolumn{3}{c}{ Year } \\
\cline { 2 - 5 } & \multicolumn{1}{c}{2011} & 2012 & 2013 & 2014 \\
\hline Workers [n] & 400 & 372 & 326 & 349 \\
$\quad$ total in year & 165 & 34 & 17 & 79 \\
$\quad$ new employees & & & & \\
Persons working for $>$ 1 year & 235 & 338 & 309 & 270 \\
$\quad$ total [n] & $183(77.9)$ & $277(82.0)$ & $263(85.1)$ & $195(72.2)$ \\
$\quad$ persons undergoing NCS testing [n (\%)] & 366 & 554 & 526 & 390 \\
$\quad$ extremities tested by NCS [n] & & & & \\
\hline
\end{tabular}

NCS - nerve conduction studies.

Table 2. Occurrence of carpal tunnel syndrome (CTS) as an occupational disease in respondents at risk for repetitive overuse of the upper extremities - working in automotive plant, Czech Republic, 2008-2014

\begin{tabular}{lrrrrrrr}
\hline \multirow{2}{*}{ Respondents } & \multicolumn{7}{c}{ Year } \\
\cline { 2 - 8 } & 2008 & 2009 & 2010 & 2011 & 2012 & 2013 & 2014 \\
\hline Workers (total) [n] & 196 & 257 & 258 & 400 & 372 & 326 & 349 \\
$\begin{array}{l}\text { CTS cases recognized as an occupational } \\
\text { disease [n] }\end{array}$ & 0 & 3 & 5 & 3 & 1 & 0 & 1 \\
\hline
\end{tabular}

Table 3. Prevalence of median neuropathy in individual years in respondents at risk for repetitive overuse of the upper extremities working in automotive plant longer than 1 year, Czech Republic, 2011-2014

\begin{tabular}{lcccc}
\hline \multirow{2}{*}{ Tested extremities and NCS findings } & \multicolumn{3}{c}{ Year } \\
\cline { 2 - 5 } & 2011 & 2012 & 2013 & 2014 \\
\hline Extremities tested [n] & 366 & 554 & 526 & 390 \\
Abnormal NCS findings [n (\%)] & $67(18.3)$ & $76(13.7)$ & $55(10.5)$ & $66(16.9)$ \\
incipient neuropathy & $54(14.8)$ & $65(11.7)$ & $55(10.5)$ & $64(16.4)$ \\
advanced neuropathy & $13(3.6)$ & $11(2.0)$ & $0(0.0)$ & $2(0.5)$ \\
sensory neuropathy & $22(6.0)$ & $24(4.3)$ & $27(5.1)$ & $41(10.5)$ \\
motor neuropathy & $2(0.6)$ & $4(0.7)$ & $6(1.1)$ & $6(1.5)$ \\
sensorimotor neuropathy & $43(11.8)$ & $48(8.7)$ & $22(4.2)$ & $17(4.4)$ \\
\hline
\end{tabular}

NCS - nerve conduction studies.

Table 3 presents the prevalence of abnormalities detected by upper extremity NCS in workers employed in the plant for at least 1 year, including their severity and type. In 2011, median neuropathy was present in $18.3 \%$ of extremities in workers employed for over 1 year. The rate steadily decreased until 2013 when only $10.5 \%$ of NCS findings were abnormal. Thus, the prevalence of median neuropathy significantly decreased in 2013 as compared 


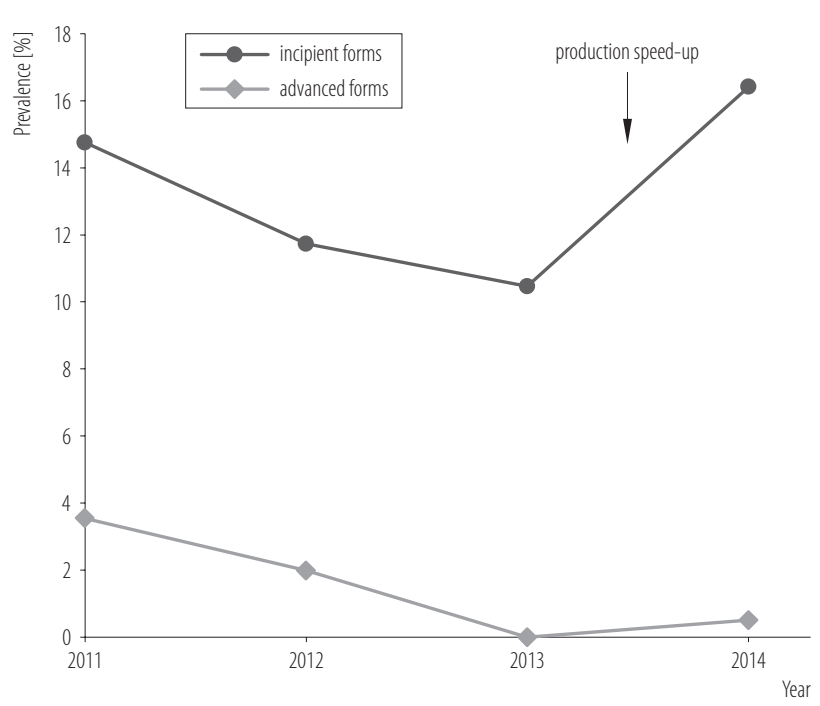

Fig. 1. Prevalence of median neuropathy of various severity in individual years in respondents at risk for repetitive overuse of the upper extremities - working in automotive plant longer than 1 year, Czech Republic, 2011-2014, expressed as a percentage from the total number of examinations

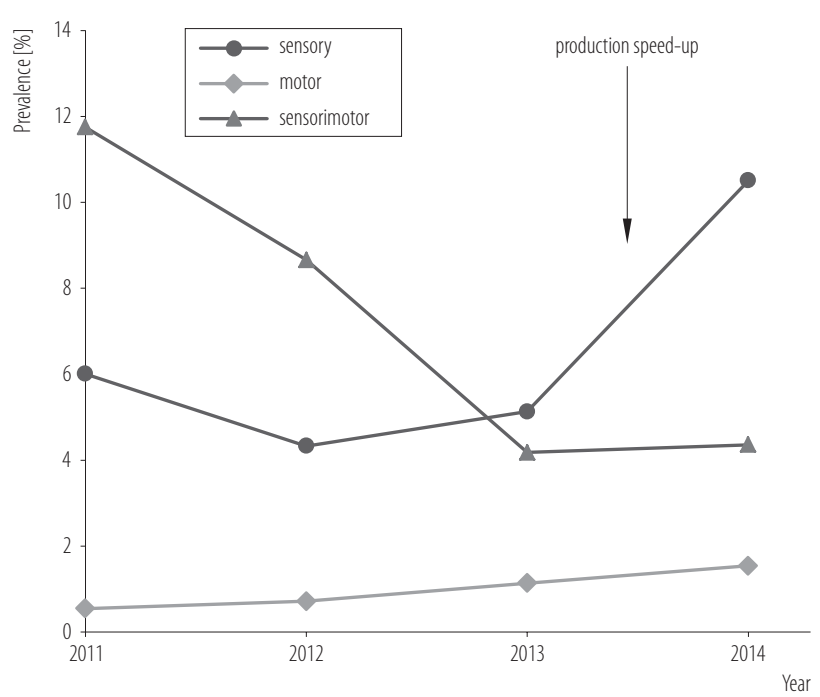

Fig. 2. Proportions of median neuropathy types in individual years in respondents at risk for repetitive overuse of the upper extremities - working in automotive plant longer than 1 year, Czech Republic, 2011-2014

with 2011 ( $p=0.003)$. In early 2014, the production pace increased and the change was accompanied by a rise in the prevalence of abnormal NCS findings to $16.9 \%$.
Figure 1 shows trends in the prevalence of advanced and incipient forms, with the latter being more common. Figure 2 presents trends in the prevalence of various forms of neuropathy, with the proportion of sensorimotor neuropathy decreasing in favor of only sensory neuropathy which was most prevalent from 2013 onwards.

There was a significant decrease in termination of employment due to detected median neuropathy, suggesting both social and economic benefits of preventive measures for the employees. In 2011, the employer terminated 13 contracts $(5.5 \%)$ due to median neuropathy among 235 workers employed for longer than 1 year. In 2014, only 1 contract $(0.4 \%)$ was terminated among 270 workers employed for longer than 1 year (Figure 3).

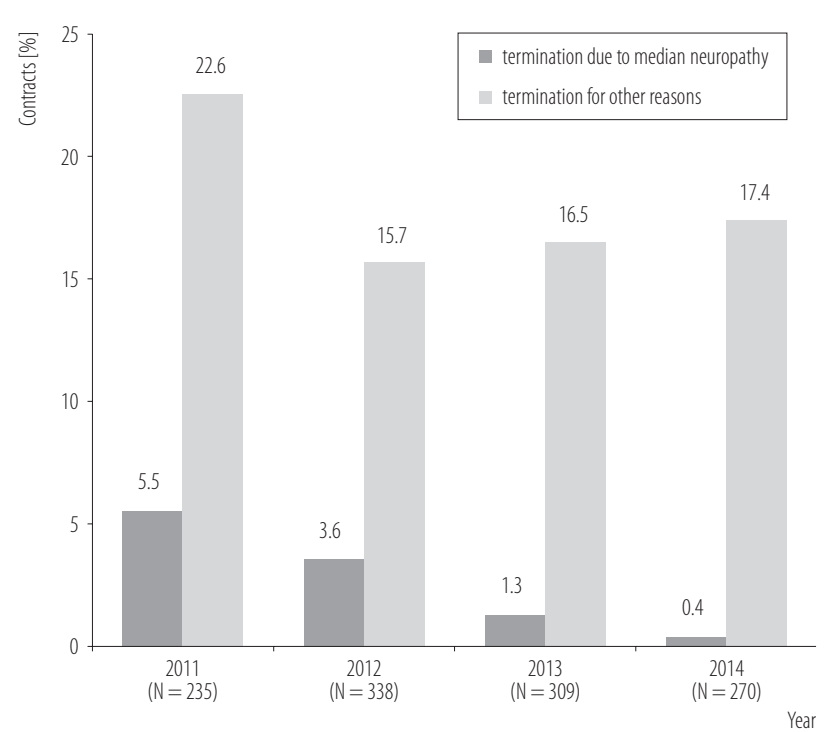

$\mathrm{N}$ - number of workers employed for longer than 1 year.

Fig. 3. Contracts terminated in Czech Republic, 2011-2014, in respondents at risk for repetitive overuse of the upper extremities - working in automotive plant longer than 1 year

\section{DISCUSSION}

Various definitions based on the presence of a range of symptoms, clinical signs, positive test results and electrodiagnostic abnormalities have been used in the 
literature to characterize CTS, while no international standard has been available for the diagnosis of the condition [20]. Therefore, the incidence and prevalence rates of CTS vary significantly, depending on the criteria used for its diagnosis [3]. If symptoms alone are considered, the prevalence of CTS is generally much higher than is the case when more accurate clinical and neurophysiological criteria are used [2,20,21]. According to a 2012 meta-analysis examining 87 studies (27 longitudinal and 60 cross-sectional) in the PubMed, EMBASE, Cochrane and Web of Science databases, the prevalence of CTS was $8.2 \%$ in the general population and $10.9 \%$ in working people [15].

In the Czech Republic, minimum clinical and hygiene criteria have to be met to recognize CTS due to overuse as an occupational disease. The condition has to be characterized as entrapment syndrome with clinical signs and pathological NCS findings of at least medium severity. At the same time, it has to be related to work activities that place stress on particular structures to the extent that, according to current medical knowledge, the overuse leads to the condition [17]. Therefore, the clinical criteria involve the objective diagnosis of isolated CTS including the required at least medium severity of NCS findings [7,17].

Electrophysiological findings in isolated CTS correspond with at least medium severity in the Czech Republic if the criteria 1 and 4 specified below are met, together with at least one of the criteria 2 or $3: 1$ ) median SCV to the index or middle finger (at $14 \mathrm{~cm}) \leq 38 \mathrm{~m} / \mathrm{s}$ or no response; 2) median DML (at $8 \mathrm{~cm}$ ) $\geq 5.3 \mathrm{~ms}$ or no response; 3) detection of abnormal spontaneous activity - the presence of fibrillation or positive sharp waves on needle NCS in the abductor pollicis brevis muscle in at least 2 areas, and at the same time there is no such activity in the muscles of the hand innervated by the ulnar nerve; and 4) normal ulnar DML and SCV to the little finger [7].
The hygiene criteria determine working conditions considered as unfavorable and leading to overuse. These are individually assessed in particular workplaces of the affected persons. The assessment includes muscle strength used, numbers of movements and working positions of the extremities depending on the extent of the static and dynamic components of work during an average 8-h shift [22].

Compared to nationwide statistics of occupational disease in the Czech Republic, CTS due to overuse was determined in younger individuals. In 2014, the median age at which the condition was recognized as an occupational disease in the Czech Republic was 47 years [14]. In the present study, however, the subjects' mean age was 44 years. Work-related CTS as an occupational disease occurred in the automotive plant after an exposure time shorter by 2.8 years as compared with the nationwide median which was 5 years in 2014 [14]. Please note that while the incidence of occupational diseases has generally decreased in the Czech Republic over the last 20 years, the trend is opposite for the automotive industry, where CTS due to overuse is the most frequently recognized occupational disease [23]. This makes the preventive measures even more necessary.

Introduction of preventive measures decreased the prevalence of median neuropathy from $18.3 \%$ of examined extremities in 2011 to $10.5 \%$ in 2013 ( $p=0.003$ ). Over the study period, the rate of sensorimotor neuropathy decreased in favor of only sensory neuropathy which has been most frequent since 2013. The percentage of employees whose contracts were terminated due to median neuropathy decreased gradually from 5.5\% to $0.4 \%$. Targeted prevention of work-related CTS is effective, as evidenced by the decrease in the prevalence of median neuropathy detected by NCS.

Many studies have been published on the etiology and risk factors for the development of CTS as well as its prevalence in various groups of people. Despite 
the fact that the need for preventive measures is frequently stressed, only few studies have assessed their effectiveness. A literature search performed by Italian authors in 2008 selected 41 studies in the Medline and the Cochrane Collaboration Occupational Health Field biomedical database on primary prevention of upper limb work-related musculoskeletal disorders. The majority investigated a wide range of general preventive interventions, but lacked well-defined risk assessment and outcome measures. Only 4 studies examined the effect of preventive measures in specific pathologies, namely CTS and hand-arm vibration syndrome. The authors conclude that only long-term studies with welldefined risk assessment and outcome measures could be used as the basis for policy recommendations [24]. In their descriptive retrospective study in the region of Massachusetts, USA, Wellman et al. [25] noted preventive measures in various employees with work-related CTS. These interventions included training in the causes and prevention of CTS and, similar to the plant in the present study, also changes of tools, switching of working tasks and thus reducing repetitive wrist movements, transfer to less demanding job or introducing more breaks [25].

Giampaoletti et al. [26] reported that after preventive measures had been introduced in companies where employees were exposed to biomechanical overload of their arms, the prevalence of CTS clearly decreased, consistent with the present study. The authors investigated 448 assembly line workers in 7 metal-mechanical plants over a period of 5 years. The preventive measures comprised decrease of repetitive wrist movements, reduction in the use of vibrating instruments and manual labor, more work breaks and an increment in position switch following the appearance of the disorders [26].

Similarly, a 5-year study carried out in the upholstered furniture industry in southern Italy examining more than 6000 filling preparation workers, leather-cutting operators and sewing and upholstery-assembly workers exposed to repetitive strain and movements of the upper limbs showed that adequate ergonomic adjustments to the workplace played a role in decreasing the upper extremity overuse [27].

Also the present study is unable to tell exactly how effective individual preventive measures were. However, it is certain that in the plant as a whole, the prevalence of work-related CTS and more severe forms of sensorimotor neuropathy has decreased, as objectively confirmed by repeated NCS testing of the median nerve. The set of preventive measures definitely played a decisive role in the trend. The results have clearly shown how sensitively the prevalence of median neuropathy reacts, for instance, to a production speed-up. Despite the improved situation, novel solutions need to be sought. Recently, selfadministered preventive kinesiotherapy of CTS and systemic enzyme therapy have been implemented by the company; their effectiveness will be prospectively studied. The authors suggest that NCS tests of the median nerve are obligatory not only during pre-employment and exit medical examinations in persons at risk for repetitive overuse, as stated in the Czech legislature since 2013 [28], but also as a part of periodic examinations, allowing a flexible response to the findings as well as a reliable assessment of the effectiveness of preventive measures that have been implemented.

The authors considered benefit of the study in exact evaluation of median nerve neuropathy based on electrophysiological testing exactly according to specified methodology. In the future, this may contribute to standard evaluation of the effectiveness of preventive measures, as this may be the only rational basis for justification of particular preventive interventions.

Additionally, the authors are convinced that for clear, obligatory and effective evidence-based preventive recommendations for CTS, an internationally acceptable standard approach to the diagnosis of this condition, accurate risk assessment including occupational risk factor 
measurements (frequency of movements, muscle strength used, etc.), clear definitions of individual preventive measures and long-term monitoring of their effectiveness are needed.

\section{CONCLUSIONS}

Carpal tunnel syndrome due to overuse is the most frequently recognized occupational condition in the Czech Republic. The same is true for the automotive plant. The active approach of an occupational physician, the company's health and safety-at-work specialist and the company management has resulted in the introduction of targeted prevention of CTS. This has been successful in that the prevalence of median neuropathy has decreased, with only incipient neuropathy being dominant.

\section{REFERENCES}

1. Lewańska M, Walusiak-Skorupa J. [Etiological factors of carpal tunnel syndrome in subjects occupationally exposed to monotype wrist movements]. Med Pr. 2014;65(2):261-70, https://doi.org/10.13075/mp.5893.2014.027. Polish.

2. Giersiepen K, Spallek M. Carpal tunnel syndrome as an occupational disease. Dtsch Arztebl Int. 2011 Apr;108(14): 238-42, https://doi.org/10.3238/arztebl.2011.0238.

3. Aroori S, Spence RA. Carpal tunnel syndrome. Ulster Med J. 2008 Jan;77(1):6-17.

4. Nordstrom DL, DeStefano F, Vierkant RA, Layde PM. Incidence of diagnosed carpal tunnel syndrome in a general population. Epidemiology. 1998 May;9(3):342-5, https:// doi.org/10.1097/00001648-199805000-00021.

5. Roquelaure Y, Ha C, Pelier-Cady MC, Nicolas G, Descatha A, Leclerc A, et al. Work increases the incidence of carpal tunnel syndrome in the general population. Muscle Nerve. 2008 Apr;37(4):477-82, https://doi.org/10.1002/mus.20952.

6. Žídková V, Nakládalová M, Straková V, Nakládal Z, Kollárová H. [Conditions for recognizing carpal tunnel syndrome from overuse as an occupational disease]. Prakt Lek. 2014;94(5):230-4. Czech.

7. [Procedure and conditions of electrophysiological testing to determine the degree of seriousness of isolated CTS]. Bull Ministry Health. 2003 Oct;10. Czech.

8. Roquelaure Y, Mariel J, Dano C, Fanello S, PenneauFontbonne D. Prevalence, incidence and risk factors of carpal tunnel syndrome in a large footwear factory. Int J Occup Med Environ Health. 2001;14(4):357-67.

9. Abichandani S, Shaikh S, Nadiger R. Carpal tunnel syndrome - An occupational hazard facing dentistry. Int Dent J. 2013 Oct;63(5):230-6, https://doi.org/10.1111/ idj.12037.

10. Barcenilla A, March LM, Chen JS, Sambrook PN. Carpal tunnel syndrome and its relationship to occupation: A meta-analysis. Rheumatology (Oxford). 2012 Feb;51(2):250-61, https://doi.org/10.1093/rheumatology/ker108.

11. Bongers FJ, Schellevis FG, van den Bosch WJ, van der Zee J. Carpal tunnel syndrome in general practice (1987 and 2001): Incidence and the role of occupational and non-occupational factors. Br J Gen Pract. 2007 Jan;57(534):36-9.

12. Maghsoudipour M, Moghimi S, Dehghaan F, Rahimpanah A. Association of occupational and non-occupational risk factors with the prevalence of work related carpal tunnel syndrome. J Occup Rehabil. 2008 Jun;18(2):152-6, https:// doi.org/10.1007/s10926-008-9125-4.

13. Palmer KT, Harris EC, Coggon D. Carpal tunnel syndrome and its relation to occupation: A systematic literature review. Occup Med (Lond). 2007 Jan;57(1):57-66, https://doi.org/10.1093/occmed/kql125.

14. National Institute of Public Health [Internet]. Prague: The Institute [cited 2015 Sep 1]. [Occupational diseases in the Czech Republic]. Available from: http://www.szu. cz/publikace/data/nemoci-z-povolani-a-ohrozeni-nemociz-povolani-v-ceske-republice. Czech.

15. Spahn G, Wollny J, Hartmann B, Schiele R, Hofmann GO. [Meta-analysis for the evaluation of risk factors for carpal tunnel syndrome (CTS). Part II. Occupational risk fac- 
tors]. Z Orthop Unfall. 2012 Oct;150(5):516-24, https://doi. org/10.1055/s-0032-1315346. German.

16. [Regulation No. 128/1975 Coll., implementing the Social Security Act, Annex 1. Collection Laws 1975, No. 30. p. 70757]. Czech.

17. [Government Decree No. 290/1995 Coll., laying down the list of occupational diseases. Collection Laws 1995, No. 76. p. 3969-78]. Czech.

18. [Regulation of the Czech Ministry of Health No. 432/2003 Coll., laying down the conditions for dividing jobs into categories, limit values of biological exposure tests, sampling conditions of biological material for biological exposure tests and requirements for reporting work with asbestos and biological agents. Collection Laws 2003, No. 142. p. 721023]. Czech.

19. [Act No. 309/2006 Coll., on other conditions of safety and health at the workplace. Collection Laws 2006, No. 96. p. 3789-97]. Czech.

20. Macfarlane GJ. Identification and prevention of workrelated carpal-tunnel syndrome. Lancet. 2001 Apr 14; 357(9263):1146-7, https://doi.org/10.1016/S0140-6736(00) 04382-8.

21. Thiese MS, Gerr F, Hegmann KT, Harris-Adamson C, Dale AM, Evanoff B, et al. Effects of varying case definition on carpal tunnel syndrome prevalence estimates in a pooled cohort. Arch Phys Med Rehabil. 2014 Dec;95(12):2320-6, https://doi.org/10.1016/j.apmr.2014.08.004.
22. [Government Decree No. 361/2007 Coll., laying down conditions for the protection of health at work. Collection Laws 2007, No. 111. p. 5086-229]. Czech.

23. Jarolímek J, Urban P. Twenty year development of occupational diseases in the Czech Republic: Medical and geographical aspects. Cent Eur J Public Health. 2014 Dec;22(4):251-6, https://doi.org/10.21101/cejph.a4063.

24. Bonfiglioli R, Farioli A, Mattioli S, Violante FS. [Evidence-based prevention and upper limb work-related musculoskeletal disorders]. G Ital Med Lav Ergon. 2008 JulSep;30(3 Suppl):26-31. Italian.

25. Wellman H, Davis L, Punnett L, Dewey R. Work-related carpal tunnel syndrome (WR-CTS) in Massachusetts, 19921997: Source of WR-CTS, outcomes, and employer intervention practices. Am J Ind Med. 2004 Feb;45(2):139-52, https://doi.org/10.1002/ajim.10326.

26. Giampaoletti C, Pisciottano V, Colao AM. [Biomechanical overload of the arm: Experience with risk prevention in the Fabrian metal-mechanical industry]. G Ital Med Lav Ergon. 2006 Apr-Jun;28(2):184-6. Italian.

27. Di Leone G, Carino M, Nicoletti S, Trani G, Ambrosi L. [Manufacture of upholstered furniture and work-related upper limb musculoskeletal disorders: An industrial sector prevention project]. Med Lav. 2008 Jul-Aug;99(4):250-61. Italian.

28. [Regulation No. 79/2013 Coll., on occupational health services and some types of medical assessment services. Collection Laws 2013, No. 37. p. 778-851]. Czech.

This work is available in Open Access model and licensed under a Creative Commons Attribution-NonCommercial 3.0 Poland License - http://creativecommons.org/ licenses/by-nc/3.0/pl/deed.en. 